\title{
Estudio endoscópico de la anatomía del seno esfenoidal en cadáveres
}

\section{Endoscopic study of the anatomy of the sphenoid sinus in cadavers}

\author{
Daniel Cantero $\mathbf{C}^{1}$, Jaime Osorio $\mathbf{M}^{1}$, Maritza Rahal $\mathbf{E}^{1}$, Claudio Campos $\mathbf{C}^{2}$, Laura Segovia $\mathbf{G}^{3}$, \\ Felipe Cardemil M'1.
}

\begin{abstract}
RESUMEN
Introducción: El seno esfenoidal presenta una relación íntima con diversas estructuras nobles de la base del cráneo.

objetivo: El objetivo de este estudio es describir la anatomía del seno esfenoidal utilizando la vía endoscópica transnasal directa.

Material y método: Médicos del Servicio de Otorrinolaringología del Hospital Barros Luco Trudeau realizaron las disecciones en cadáveres del Servicio de Anatomía Patológica de la misma institución. Se realizaron diversas mediciones de la fosa nasa en relación al seno esfenoidal y se observaron la características del seno esfenoidal en relación a las estructuras neurovasculares que se proyectan en la pared.

Resultados: Se estudiaron veinte cadáveres con una mediana de edad de 70 años. El $65 \%$ eran de sexo masculino. El 65\% presentó configuración selar. El $60 \%$ de los senos presentaron múltiples septos. El ostium esfenoidal se encontró en la mayoría de los casos medial al cornete superior. Se apreció proyección en el seno del $80 \%$ de carótidas (con 5\% de dehiscencias), $85 \%$ de nervios ópticos, $40 \%$ de nervios maxilares, $15 \%$ de nervios vidianos, y $15 \%$ de celdas de Onodi.

Conclusiones: El seno esfenoidal es una estructura compleja relacionada con estructuras nobles que se proyectan al interior de éste, por lo que su conocimiento acabado es fundamental para su abordaje quirúrgico y de la base de cráneo.
\end{abstract}

Palabras clave: Seno esfenoidal, anatomía, endocopía.

\begin{abstract}
Introduction: The sphenoidal sinus has an intimate relationship with critical structures and their exposure in the sinus walls depend on the pneumatization degree.

Aim: The aim of this study is to describe the anatomy of the sphenoid sinus using direct transnasal endoscopic approach on a sample of adult corpses.

Material and method: We performed endoscopic dissection of twenty corpses of the Pathology Department of the Hospital Barros Luco Trudeau. Various measurements were made from the nostrils. A description of the neuro-vascular structures projected into the sinus was performed.
\end{abstract}

\footnotetext{
Médico del Servicio de Otorrinolaringología, Hospital Barros Luco Trudeau-Universidad de Chile.

Médico del Servicio de Neurocirugía, Hospital Barros Luco Trudeau-Universidad de Chile.

Médico del Servicio de Anatomía Patológica, Hospital Barros Luco Trudeau-Universidad de Santiago de Chile.
} 
Results: The median age was 70 years. $65 \%$ were male. $65 \%$ of the sinuses had sellar configuration. $60 \%$ of the sinuses had multiple septa. The sphenoid ostium was in most cases located medial to the superior concha. $80 \%$ of the carotid arteries were projected into the sinuses (5\% were dehiscent). $85 \%$ of the optic nerves, $40 \%$ of maxillary nerves, and $15 \%$ of vidian nerves were observed in the sinuses. Onodi cells were found in $15 \%$ of the sinuses.

Conclusions: The sphenoid sinus is a complex structure adjacent to delicate structures. It is essential to be aware of these structures and their anatomical variations to perform a safe surgical approach of the sphenoid sinus and skull base.

Key words: Sphenoidal sinus, anatomy, endoscopic.

\section{INTRODUCCIÓN}

El hueso esfenoidal es una estructura ósea compleja y medial. Se articula con los huesos frontal, cigomático y etmoidal para constituir parte de la base del cráneo anterior y las órbitas' ${ }^{1}$.

Los senos esfenoidales ya están presentes en el feto como una prolongación de la cavidad nasal. Con un tamaño mínimo al nacimiento, se hallan plenamente desarrollados en la adolescencia ${ }^{1}$.

La neumatización es muy variable, lo que modifica la forma y tamaño de los senos, existiendo diferencias entre los lados derecho e izquierdo en un mismo hueso. En su interior protruyen la silla turca, la arteria carótida intracavernosa y los nervios ópticos, maxilar, y vidiano (Figuras 1, 2 y 3); las habituales variaciones de forma y tamaño establecen diferentes relaciones entre estas estructuras, por lo que las mismas deben ser reconocidas previamente a fin de evitar una lesión durante una cirugía ${ }^{1,2}$.

Desde principios del siglo XX la anatomía del seno esfenoidal ha sido bastante estudiada. Congodo en 1920 y Cope en 1927, y posteriormente en la década de los sesenta y setenta por Bergland (1968); Renn (1975); y Fujii (1979), establecieron las bases para el abordaje del seno esfenoidal con microscopio ${ }^{3}$.

Cuando el endoscopio fue introducido para realizar la cirugía de tumores de hipófisis hace 20 años ${ }^{3}$, nuevamente la anatomía del seno esfenoidal cobró importancia, debido a la gran variación anatómica del seno esfenoidal y su relación con estructuras nobles, debiendo conocer sus características y sus variantes a través de esta nueva tecnología para evitar complicaciones potencialmente fatales ${ }^{3}$.
No existen estudios en la población chilena que midan la prevalencia y el tipo de variaciones anatómicas endoscópicas del seno esfenoidal y estructuras adyacentes.

\section{OBJETIVO}

El objetivo del presente estudio fue describir la anatomía del seno esfenoidal en relación al abordaje transesfenoidal de la hipófisis, utilizando la vía endoscópica transnasal directa.

\section{MATERIAL Y MÉTODO}

Se llevó a cabo un estudio de corte transversal durante el período 2009-2010 en el Servicio de Anatomía Patológica del Complejo Asistencial Barros Luco Trudeau. Se realizó la disección quirúrgica endoscópica del seno esfenoidal en cadáveres que iban a ser sometidos a autopsia, mayores de 15 años, y sin antecedentes de trauma facial. Previo al procedimiento se solicitó la firma del consentimiento informado por parte del familiar más cercano, el cual se diseñó especialmente para este propósito. El estudio contó con la aprobación del Comité de Ética del Servicio de Salud Metropolitano Sur.

Los procedimientos fueron realizaron por los médicos contratados y en formación del Servicio de Otorrinolaringología del Hospital Barros Luco siguiendo el siguiente protocolo: Se dispuso el cadáver en posición supina, con el tronco y cabeza elevada a 30 grados. El cornete medio y superior fueron desplazados hacia lateral si no permitían una buena visualización. Se introdujo la óptica de $30^{\circ}$ endonasal de $4 \mathrm{~mm}$, por el piso nasal hasta el 
límite posterior cavidad nasal, visualizando y alcanzado el ángulo esfenoetmoidal. Se identificó el ostium esfenoidal ampliándolo inferior y medialmente. Se realizó la esfenoidotomía del seno derecho y luego del izquierdo, resecándose $1 \mathrm{~cm}$ de tabique posterior.

Las variables analizadas fueron edad, sexo, distancia desde el ostium esfenoidal hasta la parte anterior del cornete superior y a la espina nasal anterior, distancia del borde superior de la coana a la espina nasal y al ostium del esfenoides, grado de neumatización del seno esfenoidal, cantidad de tabiques, ubicación del ostium esfenoidal, impresión o dehiscencia de la arteria carótida interna, nervio óptico, nervio maxilar, nervio vidiano, y presencia de celda de Onodi3.

Los datos obtenidos fueron tabulados en el programa SPSS versión 15. La variables continuas fueron descritas como mediana y rango intercuartil (RIC), y las categóricas como frecuencia absoluta y relativa. Se utilizó test exacto de Fisher para el análisis según sexo, considerándose alfa de $5 \%$.

\section{RESULTADOS}

Se estudiaron veinte cadáveres con una mediana 70 años y un RIC entre 59-80 años. El rango mínimo fue 41 años, y el máximo 93 años. El 65\% de los especímenes fueron de sexo masculino.

La mediana encontrada entre ostium esfenoidal a la cabeza del cornete fue de $9 \mathrm{~mm}$ (7-10) en el lado derecho y $10 \mathrm{~mm}$ (8-11) en el lado izquierdo. La mediana encontrada entre ostium esfenoidal a la espina nasal fue de $70 \mathrm{~mm}$ (65-72) en el lado derecho y $70 \mathrm{~mm}$ (66-73) en el lado izquierdo. La mediana encontrada entre el borde superior de la coana a la espina nasal fue de $70 \mathrm{~mm}$ (67-76) en el lado derecho y $70 \mathrm{~mm}$ (66-77) en el lado izquierdo.
La mediana encontrada entre el ostium esfenoidal al borde superior de la coana fue de $23 \mathrm{~mm}$ (2225) en el lado derecho y $24 \mathrm{~mm}$ (22-27) en el lado izquierdo (Tabla 1).

El grado de neumatización del seno esfenoidal encontrado fue $65 \%$ selar, $30 \%$ preselar, y $5 \%$ conchal, sin diferencia significativa según sexo (Tabla 2). El 60\% de los cadáveres presentaban múltiples septos, sin diferencia según sexo (Tabla 3). La ubicación del ostium esfenoidal se encontraba en el $100 \%$ de los casos medial al cornete superior en el lado derecho, y $90 \%$ en la misma localización del lado izquierdo.

En el 80\% se observó impresión de la carótida en el seno, con un caso de dehiscencia de la pared, lo que representa el $5 \%$ de los especímenes estudiados. En el $85 \%$ se observó la impresión del nervio óptico. En el 40\% se observó la impresión del nervio maxilar en la pared lateral. En el $15 \%$ en el piso del seno se observa la impresión del nervio vidiano. Las celdas de observaron en $15 \%$ de los casos en forma asimétrica.

\section{DISCUSIÓN}

Los resultados encontrados en nuestro estudio son comparables con la literatura internacional. En ésta se encuentra que el patrón de neumatización, así como la proyección de las estructuras neurovasculares en las paredes del seno son variables dependiendo de la población estudiada y el método de estudio, ya sea por imágenes 0 disecciones en 4-12 (Tabla 4).

La mayoría de los senos estudiados presentaron buena neumatización, al igual que los encontrados por Lang ${ }^{1}$, Sareen ${ }^{6}$, Elwany ${ }^{7}$ y Sethi ${ }^{8}$ (selar y preselar), por ende los hallazgos endoscópicos más constantes y relevantes fueron receso ópticocarotídeo, arteria carótida interna, nervio óptico,

Tabla 1. Distancia anatómicas medidas en ambas fosas nasales

\begin{tabular}{|lllll|}
\hline Distancia & $\begin{array}{l}\text { Derecho } \\
\text { Mediana }\end{array}$ & RIC & $\begin{array}{l}\text { Izquierdo } \\
\text { Mediana }\end{array}$ & RIC \\
\hline & & & & \\
Ostium al cornete superior n: 15 & $9 \mathrm{~mm}$ & $7-10 \mathrm{~mm}$ & $10 \mathrm{~mm}$ & $8-11 \mathrm{~mm}$ \\
Ostium a la espina nasal n:18 & $70 \mathrm{~mm}$ & $65-72 \mathrm{~mm}$ & $70 \mathrm{~mm}$ & $66-73 \mathrm{~mm}$ \\
Arcada a la espina nasal n: 12 & $70 \mathrm{~mm}$ & $67-76 \mathrm{~mm}$ & $71 \mathrm{~mm}$ & $66-77 \mathrm{~mm}$ \\
Ostium a la arcada n: 11 & $23 \mathrm{~mm}$ & $22-25 \mathrm{~mm}$ & $24 \mathrm{~mm}$ & $22-27 \mathrm{~mm}$ \\
\hline
\end{tabular}


Tabla 2. Grado de neumatización del seno esfenoidal según sexo

\begin{tabular}{|lll|}
\hline $\begin{array}{l}\text { Neumatización } \\
\text { del seno } \\
\text { esfenoidal }\end{array}$ & Masculino $\mathrm{n}=14$ & Femenino $\mathrm{n}=6$ \\
\hline Preselar & 5 & 1 \\
Selar & 8 & 5 \\
Conchal & 1 & 0 \\
\hline
\end{tabular}

Fisher $p=0,428$.
Tabla 3. Cantidad de septos según sexo

\begin{tabular}{|lll|}
\hline Septos & Masculino $\mathrm{n}=14$ & Femenino $\mathrm{n}=6$ \\
Múltiples & 8 & 4 \\
Único & 6 & 2 \\
\hline
\end{tabular}

Fisher $p=0,392$.

Tabla 4. Hallazgos anatómicos del seno esfenoidal según autor

\begin{tabular}{|c|c|c|c|c|c|c|c|c|c|}
\hline Autor & Año & Preselar & Selar & $\begin{array}{l}\text { Múltiples } \\
\text { septos }\end{array}$ & $\begin{array}{l}\text { Arteria } \\
\text { carótida } \\
\text { interna }\end{array}$ & $\begin{array}{l}\text { Nervio } \\
\text { óptico }\end{array}$ & $\begin{array}{l}\text { N. } \\
\text { Maxilar }\end{array}$ & $\begin{array}{l}\text { N. } \\
\text { Vidiano }\end{array}$ & Onodi \\
\hline Perondi & 2012 & $10 \%$ & $86 \%$ & & & & & & \\
\hline Tan & 2007 & & $55 \%$ & $71 \%$ & $68 \%$ & $70 \%$ & $61 \%$ & $64 \%$ & $15 \%$ \\
\hline Murta & 2002 & & & & $89 \%$ & $55 \%$ & $30 \%$ & $25 \%$ & \\
\hline Sareen & 2005 & $25 \%$ & $75 \%$ & $80 \%$ & $71 \%$ & $0 \%$ & $0 \%$ & $25 \%$ & $0 \%$ \\
\hline Elwany & 1999 & $29 \%$ & $71 \%$ & $73 \%$ & $90 \%$ & $29 \%$ & $30 \%$ & $8 \%$ & $8 \%$ \\
\hline Sethi & 1995 & $27 \%$ & $73 \%$ & & $93 \%$ & $100 \%$ & $30 \%$ & & $10 \%$ \\
\hline Elwany & 1983 & $27 \%$ & $73 \%$ & & & & & & \\
\hline Zada & $2011^{*}$ & $25 \%$ & $63 \%$ & $40 \%$ & & & & & \\
\hline Hamid & $2008^{*}$ & $21 \%$ & $76 \%$ & & & & & & \\
\hline Kazkayasi & $2005^{*}$ & & & & $4 \%$ & $9 \%$ & $30 \%$ & $3 \%$ & \\
\hline
\end{tabular}

prominencia sellar, plano esfenoidal y clivus ${ }^{4-12}$ (Figura 4).

En todos los casos se observó el ostium natural. El abordaje del ostium natural es seguro en base a Ios reparos: Cornete superior, borde superior de la coana, y septum nasal, sobre todo cuando no existe el cornete medio por cirugías anteriores. La distancias del cornete superior al ostium del seno esfenoidal fueron $10 \mathrm{~mm}$ menor a lo encontrado por Sareen $^{6}$ de $22 \mathrm{~mm}$, pero la gran mayoría de los casos el ostium fue medial al cornete superior.

La clásica distancia de 12 a $15 \mathrm{~mm}$ entre el borde superior de la coana y el ostium natural es una proyección espacial. La distancia real entre el ostium y el borde superior de la coana, resultó en todos los casos mayor a $20 \mathrm{~mm}$.

La distancia entre el ostium del esfenoides y diversos reparos anatómicos de la fosa nasal fue un poco superior a lo reportado en la literatura. Esta diferencia se debería a que los otros estudios son mediciones en especímenes abiertos, o por tomografía computarizada, y no de forma endoscópica ${ }^{4-13}$.

La proyección de la carótida interna según Murta $^{5}$, Sareen ${ }^{6}$, Elwany ${ }^{7}$ y Sethi ${ }^{8}$ es entre $71 \%$ a $90 \%$, al igual que nuestro estudio. La dehiscencia de la arteria carótida así como del nervio óptico se observa en la literatura entre el $4 \%$ y $5 \%$. En nuestro estudio las dehiscencias en las estructuras nobles intraseno fueron bajas, correspondiendo a un caso de carótida ${ }^{5-8}$. Encontramos celdillas de Onodi en el $15 \%$ de los casos $(n=3)$. En la literatura se presentan entre el $2,6 \%$ y $15 \%$ en diferentes estudios ${ }^{4-8}$.

En más de la mitad de los casos encontramos tabiques múltiples, y cuando era único no siempre se dirigía hacia el centro de la silla turca sino incluso a estructuras nobles. Por esta razón, se refuerza el concepto de que no debe ser considerado como una referencia segura ${ }^{4-8}$. 


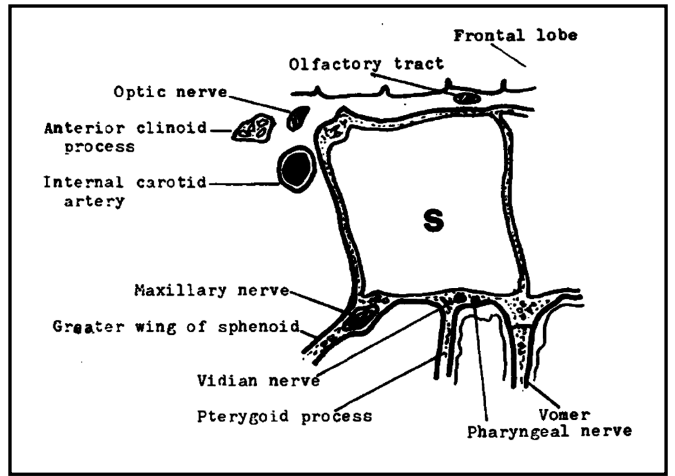

Figura 1. Corte coronal en el medio del seno esfenoidal. (Tomado de Elwany S, Yacout M, Talaat M, et col. Surgical anatomy of the sphenoid sinus. J Laryngol Otol 1983; 97: 227-41.)

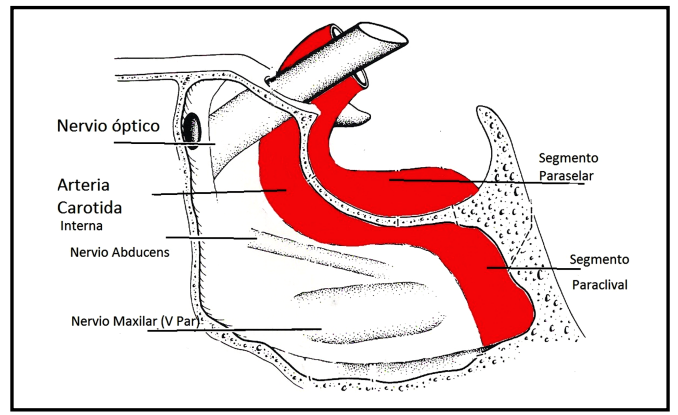

Figura 3. Corte sagital de seno esfenoidal que muestra pared lateral en relación a la arteria carótida.

La cirugía transesfenoidal de hipófisis ha ido evolucionando en la medida que han aparecido tecnologías que permiten el abordaje con el mínimo de invasión ${ }^{14-19}$. Los endoscópicos actualmente permiten una excelente visualización panorámica, gran magnificación e iluminación del esfenoides, con el mínimo de trauma, mejorando el confort para el paciente y disminuyendo la morbilidad después de la cirugía ${ }^{14-19}$. No se debe olvidar que la visión endoscópica es monocular, y los lentes gran angulares producen distorsión que se incrementa con la mayor angulación de la óptica ${ }^{14-19}$.

Por supuesto, la anatomía microscópica como endoscópica son básicamente las mismas, pero la distorsión óptica de imágenes endoscópicas es bastante sustancial en comparación con las representaciones microscópicas. Una lente del endoscopio produce imágenes con aumento máximo en su centro y contracción severa en su periferia. Imágenes más cercanas son desproporcionadamente ampliadas e

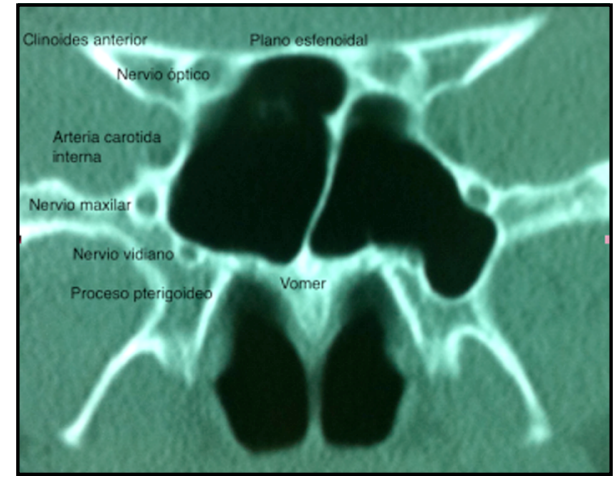

Figura 2. Tomografía computarizada corte coronal del seno esfenoidal.

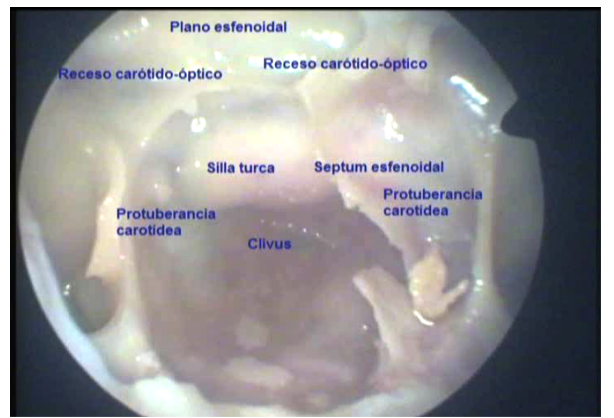

Figura 4. Pared posterior del seno esfenoidal con óptica de $30^{\circ}$ en disección endoscópica.

imágenes remotas son miniaturizadas falsamente. Esta ilusión óptica puede desorientar a un cirujano que no está familiarizado con esta condición peculiar en la base del cráne $0^{17}$.

Por último, es fundamental realizar una evaluación preoperatoria de la imágenes para planificar el abordaje y la distancia estimada con estructuras nobles, disminuyendo el riesgo de desorientación espacial y las complicaciones asociadas ${ }^{14-19}$.

\section{CONCLUSIÓN}

Este trabajo describe las relaciones anatómicas del seno esfenoidal en una población local y reafirma la complejidad de la anatomía del seno esfenoidal y las estructuras adyacentes de la base de cráneo, cuya compresión es fundamental para la práctica quirúrgica en el seno esfenoidal y como vía de abordaje de lesiones selares, paraselares y 
supraselares. Esto es importante ya que cada vez es más popular el abordaje de la silla turca y del seno esfenoidal vía transnasal directa con el uso del endoscopio en detrimento del microscopio.

Un acabado conocimiento anatómico y tomográfico de los senos esfenoidales es imprescindible a fin de aumentar la seguridad y eficiencia de la cirugía de la región selar, del clivus o del vértice orbitario, y para comprender las patologías de la región y sus complicaciones.

\section{Agradecimientos}

A todos los médicos del Servicio de Otorrinolaringología del CABLT y al personal del Servicio de Anatomía Patológica que hicieron posible este proyecto.

Trabajo financiado con fondos del concurso anual de investigación de la Sociedad Chilena de Otorrinolaringología, Medicina y Cirugía de Cabeza y Cuello, año 2009.

\section{BIBLIOGRAFÍA}

1. Lang J. Clinical anatomy of the Nose, Nasal cavity and Paranasal Sinuses. Thieme Medical Publishers. Inc. New York. 1989; 85-98.

2. Stammberger HR. Paranasal sinuses: anatomic terminology and nomenclature. The Anatomic Terminology Group. Ann Otol Rhinol Laryngol Supp/ 1995; 167: 7-16.

3. Perondi Ge, Isolan GR, de Aguiar PH, et col. Endoscopic anatomy of sellar región. Pituitary 2012; 31: 0-7.

4. TAN HK. Sphenoid sinus: an anatomic and endoscopic study in Asian cadavers. Clin Anat 2007; 20(7): 745-50.

5. Murta A. Endoscopic anatomy of the sphenoid sinus. Rev Bras Otorrinoloringol 2002; 68(4): 522-5.

6. Sareen D. Study of sphenoid sinus anatomy in relation to endoscopic surgery. Int J Morphol 2005; 23(3): 261-6.
7. Elwany S, Elsaeid I, Thabet H. Endoscopic anatomy of the sphenoid sinus. J Laryngol Otol 1999; 113 : 122-6.

8. Seth. Endoscopic anatomy of the sphenoid sinus and sella turcica. J Laryngol Oto/ 1995; 109: 951-5.

9. Elwany S, Yacout M, Talaat M, et col. Surgical anatomy of the sphenoid sinus. J Laryngol Otol 1983; 97: 227-41.

10. Zada G, Agarwalla P, Mukundan S, et col. The neurosurgical anatomy of the sphenoid sinus and sellar floor in endoscopic transsphenoidal surgery. J Neurosurg 2011; 114: 1319-30.

11. HAMID 0. Anatomic Variations of the Sphenoid Sinus and Their Impact on Transsphenoid Pituitary Surgery. Skull base 2008; 18: 9-16.

12. KazkayasI M. Anatomic variaciones of the sphenoid sinus on computed tomography. Rhinology 2005; 43: 109-14.

13. Enatsu K. Surgical anatomy of the sphenoid sinus on the CT using multiplanar reconstruction technique. Otolaryngol Head Neck Surg 2008; 138: $182-6$.

14. Cavallo LM. Extended endoscopic endonasal trasphenoidal approach to the suprasellar area: anatomic considerations- part-1. Neurosurgery 2007; 61: 24-33.

15. SetHI. Endoscopic Pituitary Surgery. Otolaryngol Clin N Am 2006; 39: 563-83.

16. Rosen M. A review of the endoscopic approach to the pituitary through the sphenoid sinus. Curr Opin Otolaryngol Head Neck Surg 2006; 14: 6-13.

17. Cappabianca. Endoscopic Endonasal Transsphenoidal Surgery. Nerosurgery 2004; 55: 33-941.

18. Olavarría C, Stott C, Lemp M, et col. Comparación de dos técnicas quirúrgicas para abordaje de la región selar: transeptal transesfenoidal versus transnasal directa. Rev Otorrinolaringol Cir Cabeza Cuello 2004; 64: 91-8.

19. Sariego H, Bogado M. Evaluación nasal en el abordaje quirúrgico de la patología selar. Rev Otorrinolaringol Cir Cabeza Cuello 2008; 68: 164-70. 